Revue des patrimoines

\title{
Du numérique au digital. Documenter et valoriser les sépultures monumentales néolithiques
}

Documenting and interpreting monumental Neolithic tombs

\section{Serge Cassen et Valentin Grimaud}

\section{OpenEdition}

\section{Journals}

Édition électronique

URL : http://journals.openedition.org/insitu/21636

DOI : 10.4000/insitu.21636

ISSN : 1630-7305

Éditeur

Ministère de la Culture

Référence électronique

Serge Cassen et Valentin Grimaud, « Du numérique au digital. Documenter et valoriser les sépultures monumentales néolithiques », In Situ [En ligne], 39 | 2019, mis en ligne le 28 mai 2019, consulté le 10 décembre 2020. URL : http://journals.openedition.org/insitu/21636 ; DOI : https://doi.org/10.4000/ insitu. 21636

Ce document a été généré automatiquement le 10 décembre 2020.

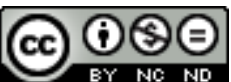

In Situ Revues des patrimoines est mis à disposition selon les termes de la licence Creative Commons Attribution - Pas d'Utilisation Commerciale - Pas de Modification 4.0 International. 


\title{
Du numérique au digital. Documenter et valoriser les sépultures monumentales néolithiques
}

\author{
Documenting and interpreting monumental Neolithic tombs
}

\section{Serge Cassen et Valentin Grimaud}

1 Nous aimerions rendre compte d'une recherche menée autour de stèles et de tombes néolithiques à travers une imagerie $2 \mathrm{D}$ et $3 \mathrm{D}$ en cours de construction. Sans revenir sur ce parcours, qui a largement bénéficié des progrès de la photogrammétrie depuis 2011, rappelons que le monument de Gavrinis (Morbihan) puis plusieurs sites de Vendée, de l'Essonne, de Bourgogne et du Valais en Suisse ont amplement contribué à l'élaboration d'une méthodologie adaptée et à la mise au point de protocoles de levés désormais appliqués à un programme collectif de recherche (PCR «Corpus des signes gravés en Bretagne »).

2 Cet inventaire s'attache à enregistrer et représenter la gravure, le signe, au même niveau que le support auquel ils sont étroitement associés, physiquement et symboliquement. La contextualisation des supports ornés, dans le cadre topographique (le site) et architectural (la tombe, l'ouvrage de stèles), s'impose tout autant afin que ne se perde pas le lien historique et fonctionnel. Les enregistrements des surfaces sont de surcroit l'occasion d'observations qui ne se limitent pas à la seule acquisition archéologique des tracés, mais relèvent les différents désordres dus aux colonisations biologiques, aux thermo-altérations, etc. On se propose ainsi d'effectuer une sorte d'état des lieux qui résume, notamment par une cartographie, cet aspect altéré du support afin d'aider à la décision autour de futures mesures conservatoires.

3 Le mode de transmission des résultats obtenus, enfin, fait partie de la réflexion qui débouchera sur des propositions concrètes, aussi bien en termes de médiation, par le biais 
d'un site dédié sur Internet, qu'en termes de stockage et d'archivage pérenne des données mises en œuvre.

4 Pour illustrer une partie de ce travail et les rendus afférents dans le cadre de ce numéro spécial d'In Situ. Revue des patrimoines, seront comparés les résultats graphiques inédits obtenus de deux ensembles monumentaux implantés sur le littoral vendéen et morbihannais. Ces constructions en élévation, qui sont une singularité propre à la préhistoire européenne entre les $\mathrm{V}^{\mathrm{e}}$ et III ${ }^{\mathrm{e}}$ millénaires av. J.-C., n'ont pas été choisies pour être les plus connues ou les plus spectaculaires. La monumentalité est une question toute relative... Elles sont pourtant représentatives de ces objets souvent nommés, par leur forme traditionnelle, «menhir » ou « dolmen ». Elles sont surtout emblématiques d'une catégorie architecturale ("sépultures mégalithiques transeptées ») bien localisée au débouché de la Loire. Les deux tombes choisies sont cependant situées aux deux extrêmes de cette distribution, l'une en Vendée à lîle d'Yeu, l'autre à Plœmeur dans le Morbihan. La première n'a jamais été restaurée, la seconde le fut dans les années 1970. L'une va disparaître sous la montée progressive des eaux, l'autre est au contraire bien ancrée dans l'imagerie armoricaine. Les deux nécessitaient d'être documentées pour discuter et comparer leurs états respectifs.

5 Pour établir cette documentation, nous avons recours, en résumé2 à :

6 - la photogrammétrie, afin de restituer la morphologie tridimensionnelle des supports, volumes dans lesquels seront rapportés les signes gravés levés.

7 - des images compilées sous éclairages obliques réels à partir de photographies, ou virtuels à partir d'une carte des reliefs, voire, dans certains cas favorables, détectés grâce à une carte des écarts.

8 - la décorrélation d'images (captation des teintes), un travail sur les espaces de couleur qui contribue à la détection des tracés mais le plus souvent à la cartographie des désordres (altérations chromatiques).

\section{La tombe à couloir de l'île d'Yeu (Vendée)}

9 Malgré un caractère insulaire déjà attesté au Néolithique moyen $(-4500,-3700)$, Yeu compte de nombreux sites dits "mégalithiques ». On y dénombre au moins 4 tombes à couloir ou à vestibule, et 8 stèles ou groupes de stèles. Augustin Auger entreprend le premier la fouille de la Planche à Puare, en 1883 ; dès 1887, le monument est classé au titre des monuments historiques. Théodore Volkov, conservateur du musée Alexandre-III à Moscou, visite l'île dans les années 1880 et publie une assez bonne description de ces dolmens ${ }^{3}$. L'exposé détaillé de la Planche à Puare est accompagné d'un dessin qui permet de constater que l'architecture n'a pas subi de dégradations significatives depuis plus d'un siècle. 
Figure 1

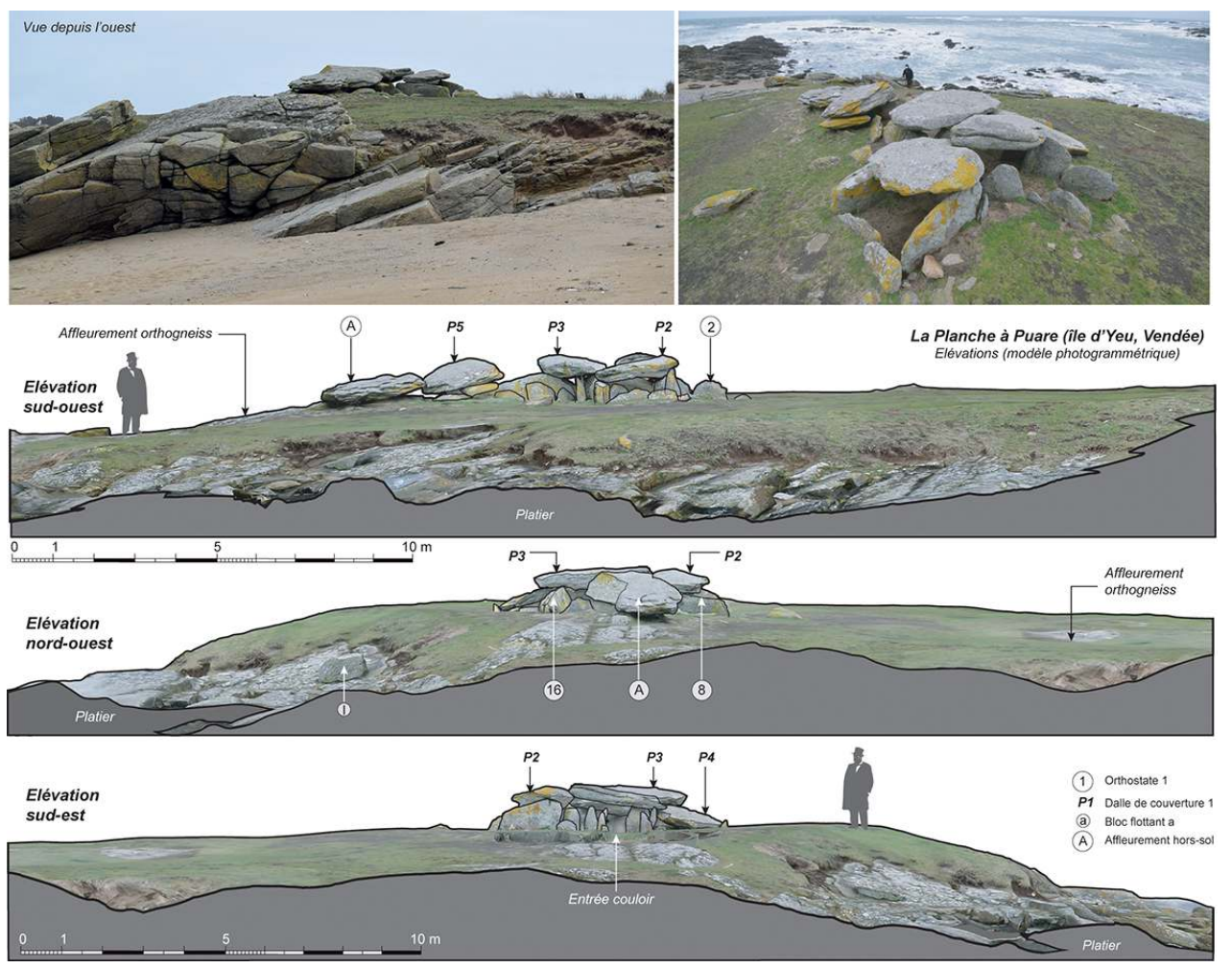

Photographies de la tombe à couloir de la Planche à Puare (Yeu, Vendée) dans son environnement marin. Les élévations extraites du modèle photogrammétrique englobent les surfaces attenantes aux structures mégalithiques internes.

(c) Serge Cassen, Valentin Grimaud.

Le monument est très proche de la bordure littorale, au point qu'une partie du cairn originel a déjà été emportée (fig. 1). La montée du niveau marin, très perceptible sur cette partie de l'île, provoquera à moyen terme la disparition du site. Le suivi précis du trait de côte nous a donc semblé un objectif à atteindre ; une fiche a donc été implantée dans le rocher pour servir de référentiel altimétrique (levé centimétrique au GPS ${ }^{4}$ différentiel). La fréquentation touristique, en hausse constante depuis vingt ans, entraîne de surcroît une dégradation des sols autour des structures internes les plus visibles (orthostates, dalles de couvertures). Les structures externes sont beaucoup plus discrètes, mais il est encore possible d'y distinguer, sous la forme de microreliefs parfois associés à des blocs de pierre, l'emprise du tumulus originel. S'il est bien difficile aujourd'hui d'apprécier à l'œil nu ces anomalies de relief, la microtopographie obtenue de la couverture photogrammétrique permet désormais de révéler ces restes périphériques (fig. 2), information utile à l'archéologue mais tout autant au visiteur de passage qui comprend mieux l'envergure du site et les parties enfouies, tout autant dignes de respect et de protection. 
Figure 2

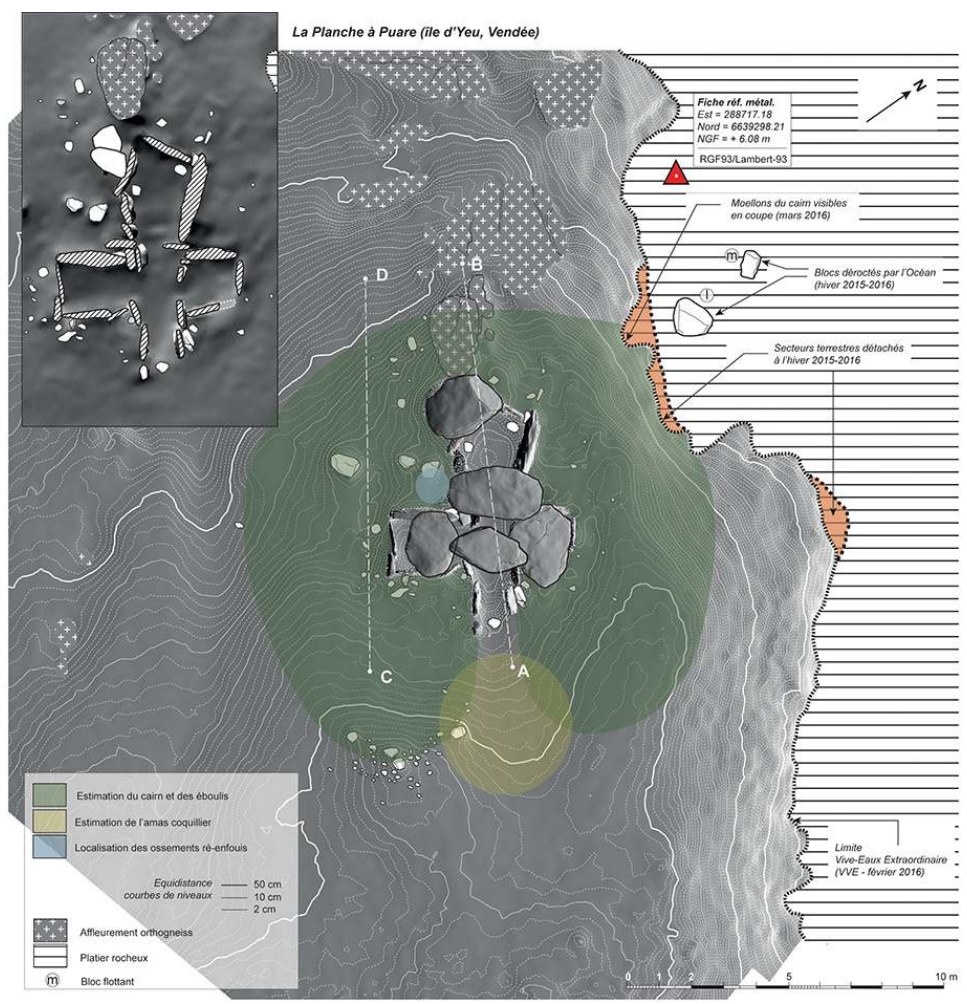

Levé photogrammétrique permettant : la microtopographie du cairn et du rivage rocheux, le plan des structures internes et leurs élévations, des vues en perspective. Le document repère les points névralgiques sur le trait de côte, et précise une des bornes servant de référentiel altimétrique.

(c) Serge Cassen, Valentin Grimaud.

La tombe est composée d'un couloir qui permet d'accéder aux trois chambres. Sa particularité réside dans le système d'encadrement des accès entre la croisée du transept et les trois cellules, qui réduit la largeur du passage. De tels rétrécissements permettaient peut-être d'obstruer complètement l'entrée avec un autre bloc. Ce fut d'ailleurs le cas pour la chambre nord où l'entrée de la cellule était «fermée par une pierre plate ». En 1883, le monument était encore peu perturbé, et contenait des ossements, une rareté sur le Massif armoricain. Leur conservation est due au PH des sols dont le sable coquillier, largement présent dans l'unité sédimentaire superficielle, a neutralisé l'acidité naturelle des horizons d'altération de l'orthogneiss.

La première partie fouillée fut la croisée du transept. À 1,30 m de la dalle de couverture, un dallage de pierres est relevé sous lequel A. Auger découvrit les ossements humains, principalement des longs os (tibias, fémurs, humérus), « rangés symétriquement, dans le sens de la longueur $»^{6}$. Aucun objet ne leur était associé. Dans la cellule latérale au sudouest, un squelette fut bien détecté, la tête au nord, « couché sur le côté droit, le bras droit replié sous la tête, le bras gauche porté en avant, les jambes recourbées et croisées... ${ }^{7}$. Des ossements d'animaux accompagnaient le corps et parmi ces derniers furent trouvées trois dents, tout d'abord considérées comme provenant d'animaux domestiques avant que Marcel Baudouin ne réalise qu'il s'agissait de dents de cachalot ${ }^{8}$, ce qui n'est pas sans intérêt s'agissant du monument que nous décrirons ensuite dans le Morbihan, où cet animal est représenté. Enfin, sous le dallage sur lequel reposait ce corps, deux autres individus furent identifiés. Dans la cellule latérale au nord-est et dans la 
chambre terminale, d'autres ossements humains étaient disposés en couches superposées, séparées par un dallage de pierres plates. Le mobilier, aujourd'hui perdu, semble s'être limité à des «pierres à filet » et des fragments de céramique. M. Baudouin estimait le nombre d'inhumés à une douzaine (dont onze mandibules). Certains os ont été travaillés post-mortem (encoches, entailles, stries, traces de grattage, etc.), majoritairement des humérus, tibias et fémurs ${ }^{9}$.

La représentation tridimensionnelle des supports et des dalles de couverture permet de produire un géométral rigoureux (plan, élévations, sections), seule manière pour que l'objet archéologique, ou l'élément d'architecture, puisse être comparé à d'autres relevés ou à d'autres objets similaires (fig. 3).

Figure 3

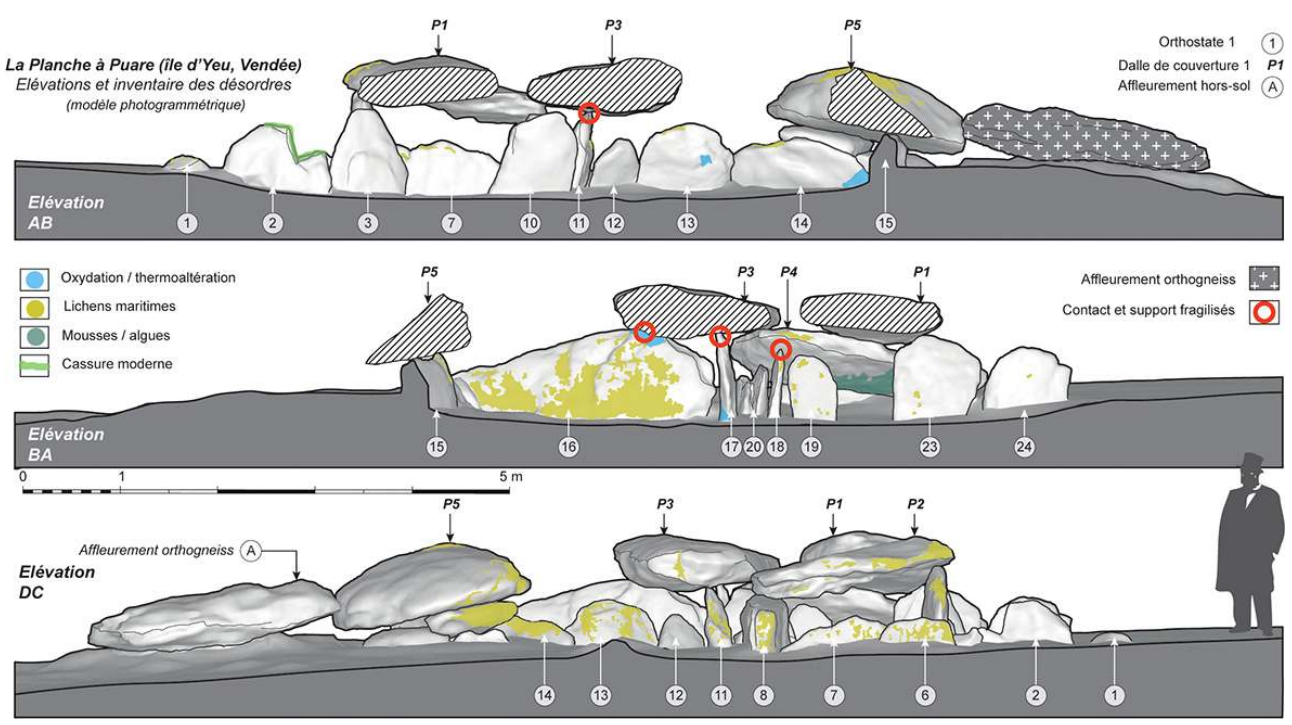

Les élévations extraites du modèle photogrammétrique permettent de graphiquement décrire les désordres affectant les structures internes.

(c) Serge Cassen, Valentin Grimaud.

Le modèle 3D rend également possible une restitution virtuelle des dépôts humains à l'intérieur des cellules, en vue planaire ou isométrique. La commune d'Yeu est d'ailleurs désireuse d'offrir une application permettant aux visiteurs de comprendre le site et son architecture; une borne interactive dans une Maison du patrimoine de l'île sera prochainement dédiée à la découverte des monuments (fig. 4). 


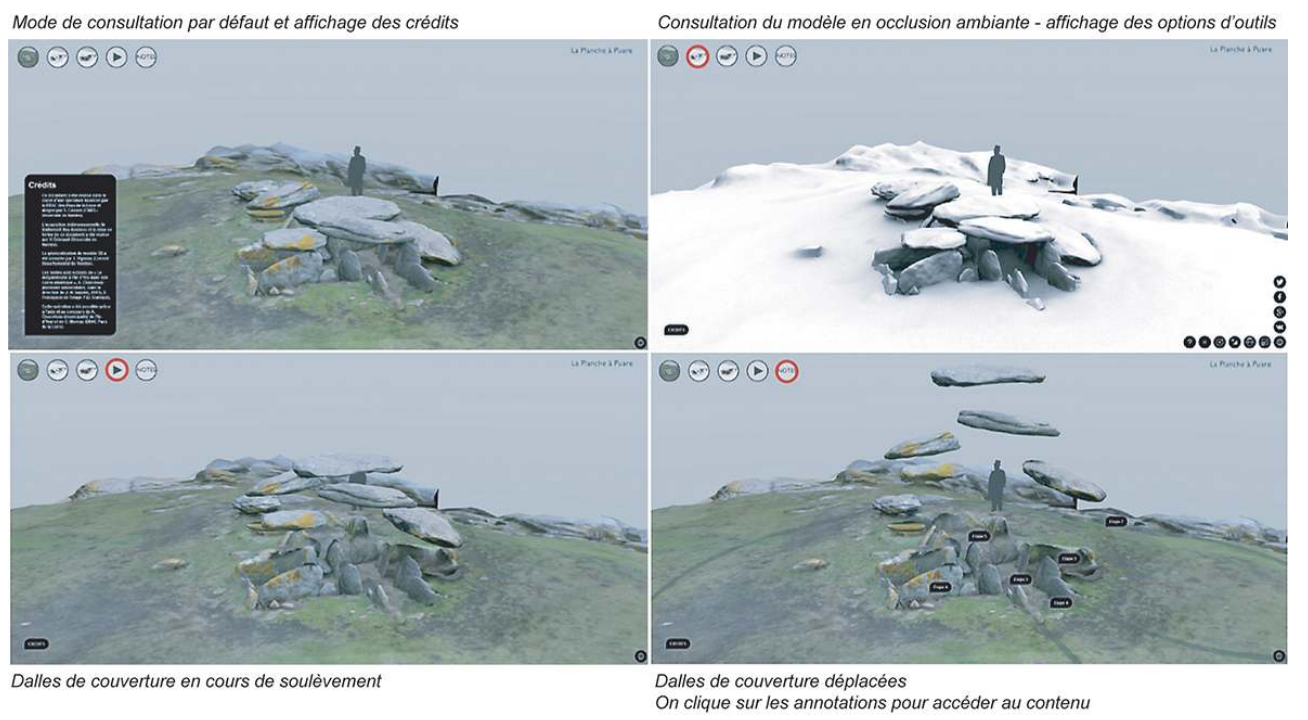

Une possibilité de consultation du modèle 3D offerte par le cadre applicatif open source Blend4Web (création et publication des infographies 3D interactives dans un navigateur web). Le visiteur peut modifier les textures et soulever les dalles de couverture pour accéder à plusieurs informations sur la fouille et l'histoire du monument (création de l'application V. Grimaud).

(c) Serge Cassen, Valentin Grimaud.

En attendant, ce support graphique donne aujourd'hui l'occasion d'établir un descriptif géomorphologique simple relatif aux orthostates et à la nature des désordres observés. Nous ne présenterons ici que sept orthostates pouvant illustrer à leur manière l'exercice en question (voir fig. 3).

$\mathrm{N}^{\circ} 11$ : Ce pilier en orthogneiss ne laisse pas d'inquiéter. Il maintient en effet la dalle P3, la plus massive des éléments de couverture, alors que la surface de contact est étroite et le support très mince, ayant probablement perdu de la matière au cours du temps (desquamation, éclats de compression).

17 № 13 : Il s'agit d'une face d'affleurement visible sur l'élévation présentée, tandis que la partie externe est clairement une face d'arrachement. Une marque réduite d'oxydation est visible sur son milieu droit.

$\mathrm{N}^{\circ} 14$ : La présentation du bloc est similaire au $\mathrm{n}^{\circ} 13$ : affleurement en paroi interne, arrachement en extérieur. Une thermoaltération couvre tout l'angle droit au contact avec le sol actuel.

$\mathrm{N}^{\circ} 16$ : Cette surface d'affleurement (une vasque est encore visible) en orthogneiss est couverte de lichens maritimes, en raison probable d'une bonne exposition au sud-ouest. Son sommet vient au contact de la dalle $\mathrm{P} 3$, mais en un point très étroit qui ne rassure nullement sur la stabilité du support. Car il est manifeste qu'un grand éclat de compression est parti sous le poids de la dalle de couverture ; le mouvement conséquent de cette dernière l'a sensiblement orienté vers le nord-est par la seule gravité. Ajoutons qu'une large tache d'oxydation marque ce secteur fragile, sans qu'il soit possible d'affirmer qu'il s'agit là d'une chauffe directe de la roche.

$\mathrm{N}^{\circ} 17$ : Cet orthostate, décidément bien mince en son sommet porteur, est marqué d'une tache d'oxydation vers le sol. $\mathrm{Au}$ nord-ouest, la face météorisée témoigne de 
l'affleurement sur l'émergence en orthogneiss, et sur l'autre côté, il en découle une face d'arrachement bien reconnaissable.

21 № 19 : Exceptionnellement, cette dalle ne paraît pas être un orthogneiss, mais nous ne saurions qualifier ce granite. Au surplus, les surfaces d'érosion sont telles (formes arrondies généralisées) qu'il nous semble très probable que le prélèvement a été effectué sur un estran rocheux. Elle est aussi penchée vers le nord-est et supporte la dalle P4.

$\mathrm{N}^{\circ} 20$ : Cette dalle posée de chant est fragilisée par le litage de l'orthogneiss. La tranche exposée aux intempéries a vu les fentes s'élargir sous l'effet de la météorisation. Elle soutient P4 qui, fort heureusement, touche le sol par ailleurs, évitant d'appuyer sur cet objet par trop fragile.

\section{Résumé conclusif}

La Planche à Puare est un bon exemple d'une tombe à couloir dite transeptée. Son tumulus est conservé sans avoir subi de restauration mais, faute d'exploration moderne, le contour en est inconnu. Le processus de détérioration de la roche est ici largement à l'œuvre, une roche souvent feuilletée et pour cette raison encline à subir plus rapidement qu'une roche compacte une météorisation dommageable. Ce défaut accentue la ruine de la structure interne, notamment au niveau de la dalle de couverture P3, en équilibre précaire sur les pointes vives des orthostates (le monument voisin des Petit Fradets a subi la fracture d'une large dalle sous l'effet d'un récent séisme de magnitude 3,7). Mais le danger le plus évident demeure, bien entendu, le recul du trait de côte, observé tempête après tempête ces dernières années. Le cairn est visible en coupe; l'angle nord-est a d'ores et déjà été emporté par l'océan. Une image est donc en train de se modifier.

\section{La tombe à couloir de Cruguellic (Ploemeur, Morbihan)}

Dominant les plages de Fort-Bloqué à l'ouest, la tombe à couloir de Cruguellic est aujourd'hui cachée derrière un rideau de végétation empêchant toute visibilité vers le large. Cette implantation sur le versant du relief, et non vers le sommet, qui est la norme la plus fréquente pour les « dolmens » de cette époque, est très probablement en rapport avec cette co-visibilité maritime voulue, à l'image des monuments du même type transepté inventoriés autour de l'embouchure de la Loire qui privilégient ces choix topographiques sur la pente dominant l'océan ou les actuels marais littoraux ${ }^{10}$.

Malmené pendant la guerre (installation d'un projecteur anti-aérien), le monument a subi de nouveaux dommages lors de la construction d'un centre de vacances, dommages qui ont finalement incité la direction des Antiquités préhistoriques de Bretagne à intervenir par la fouille, en 1974 et 1975. Les autres perturbations constatées semblent remonter aux époques gallo-romaine et médiévale. Nous l'avons dit, il s'agit de l'exemple le plus occidental des tombes de ce type, majoritairement réparties entre la région de Carnac et la Basse-Loire (fig. 5). Le cairn est quadrangulaire et limité par des muraillements en pierre sèche ${ }^{11}$. Le mobilier récolté renvoie au Néolithique moyen (ensemble régional Auzay-Sandun : 4200-3800 av. J.-C.). 
Figure 5

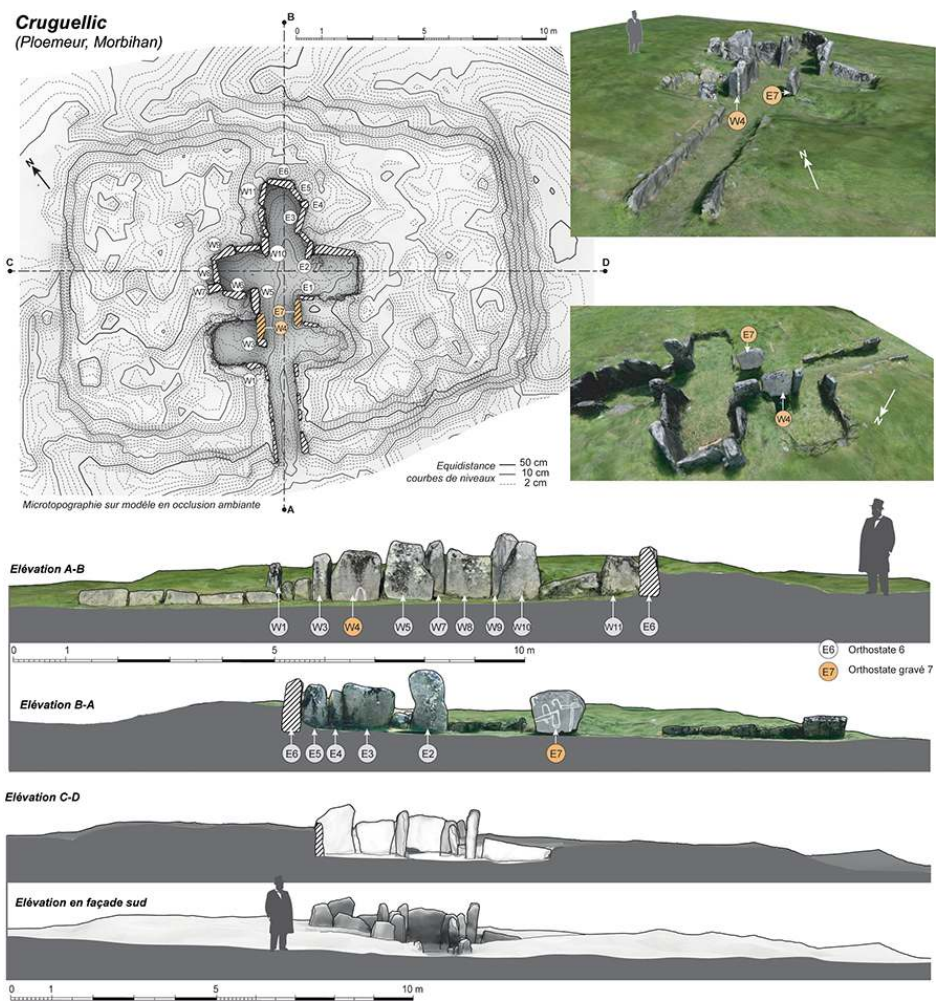

La tombe néolithique de Cruquellic (Plœmeur, Morbihan). Levé photogrammétrique permettant : la microtopographie du cairn, le plan des structures internes et leurs élévations, des vues en perspective. (c) Serge Cassen, Valentin Grimaud.

Deux des montants en granite portent une ornementation gravée (fig. 6), interprétée dans les deux cas comme des figurations dérivées de «l'écusson », motif protéiforme et ubiquiste du registre iconographique breton mis en place entre 1860 et 1930.

Figure 6
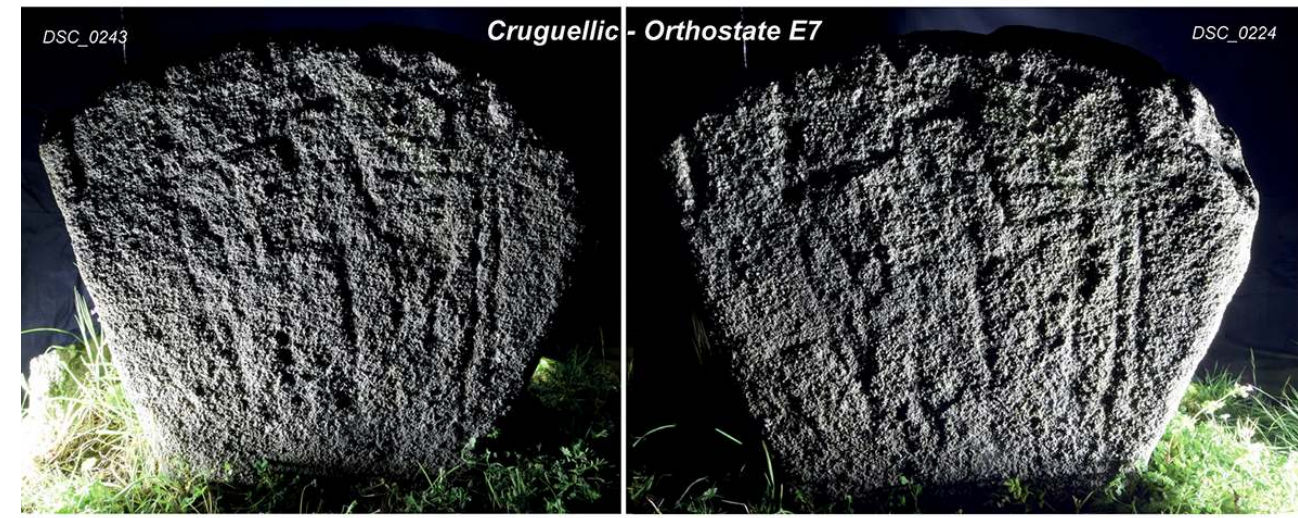

Deux clichés sur l'orthostate E7 illustrant la technique des éclairages obliques à foyers opposés.

(c) Serge Cassen, Valentin Grimaud.

La publication de 1977 décrit le bloc E7, choisi dans notre illustration, comme ayant été trouvé déchaussé et brisé sur le site ${ }^{12}$, alors qu'il était encore bien reconnaissable, debout et calé (fig. 7), sur la photographie du début des années 1970 (fig. 39 dans Le Roux, 1975). 
On note aussitôt que cette orientation de la dalle (et donc des gravures) est à l'inverse de la position actuelle restaurée; le premier levé des gravures suit par conséquent cette ancienne disposition (fig. 5 dans Le Roux, Lecerf, 1977). Les auteurs reconnaissent, parmi d'autres lignes, les restes d'un «écusson» à double encadrement et ligne médiane verticale, ornementation classique des dolmens à couloir morbihannais ${ }^{13}$.

Figure 7
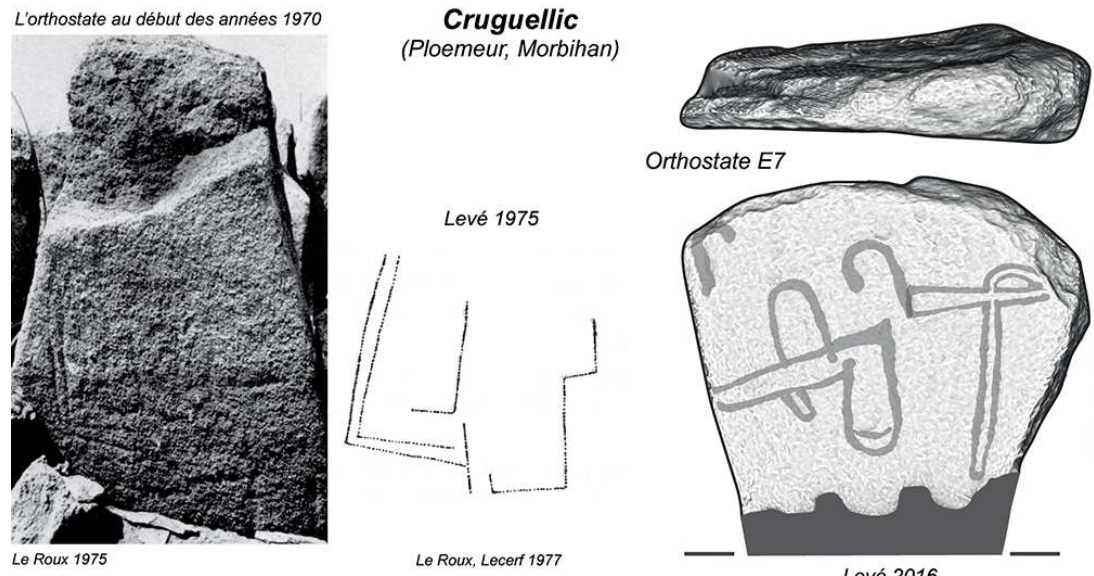

L'orthostate après la restauration

Deux positions de l'orthostate E7 : avant restauration et bouleversement des structures, à gauche ; après la restauration à droite.

Phot. C.T. Le Roux, SRA Bretagne. (c) SRA Bretagne.

\section{Procédures d'acquisition}

La morphologie tridimensionnelle des supports (fig. 8) a été rendue par photogrammétrie (orthostate E7 : 75 clichés). Une seule station par dalle fut nécessaire pour le levé des gravures, étant donné la taille réduite des supports, bien calibrés pour un capteur à 24 millions de pixels placé à $1 \mathrm{~m}$ de distance (Nikon 5300). Le dessin vectoriel des tracés et anomalies fut opéré sur tablette graphique. 


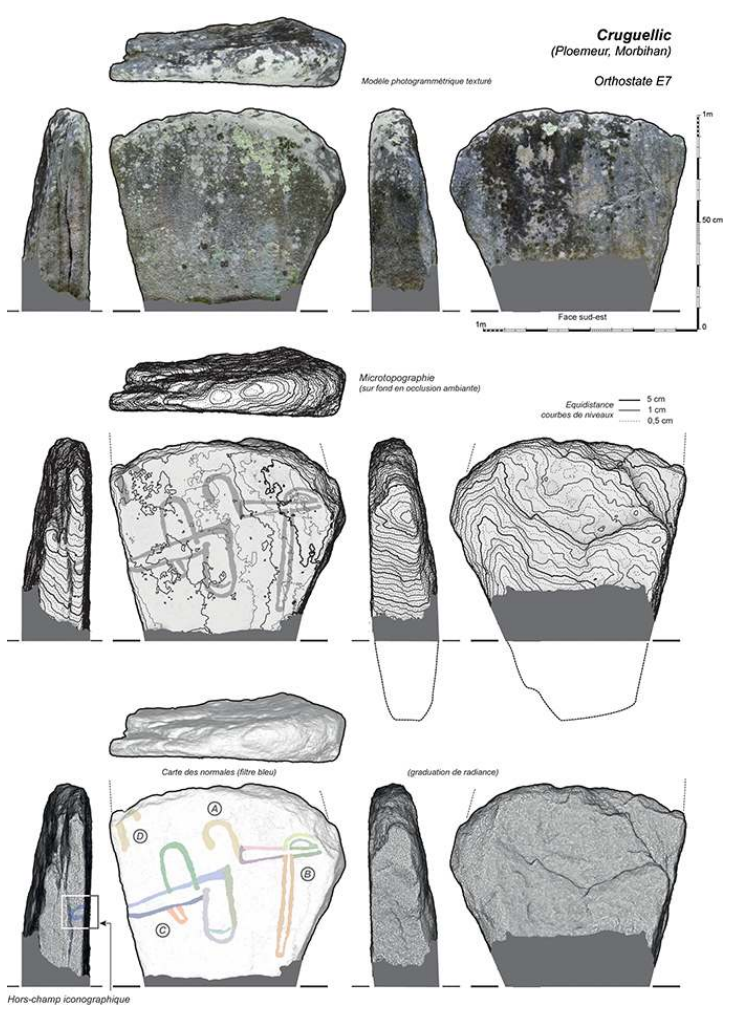

L'orthostate E7 selon trois rendus du géométral : vue photoréaliste, microtopographie des surfaces, inscription des motifs et prolongement du motif $\mathrm{C}$ sur la tranche gauche.

(c) Serge Cassen, Valentin Grimaud.

\section{Orthostate E7}

Le levé des gravures, par compilation de photographies sous éclairages tournants obliques (voir fig. 6, fig. 8), levé sur lequel porte notre descriptif, a été mené sous un barnum de toile opaque et s'est fondé sur 1 station d'acquisition (61 clichés, Nikkor $10 \mathrm{~mm}$ ). Le corpus graphique est composé de 49 fichiers vectoriels correspondant à autant de clichés choisis parmi les 61 photos réalisées.

La dalle est un granite à deux micas, sur laquelle il est presque impossible en plein jour de détecter les gravures. Seul un éclairage rasant vers midi laisse entrevoir leur présence, encore que l'observateur non averti puisse très facilement confondre certains plis naturels avec des tracés anthropiques. On est au surplus assez inquiet des difficultés actuelles à les distinguer quand on examine la dalle photographiée en $1974^{14}$ où aucune colonisation végétale n'est visible (voir fig. 7). La couverture actuelle par les lichens doit donc contribuer à cet estompage. Mais il est également vrai que la météorisation, même en quarante ans d'attaque de la roche, ce qui peut paraitre un espace de temps bien réduit, a significativement affecté la surface du support.

Trois motifs bien connus du répertoire armoricain ont été identifiés : crosses de jet, hache emmanchée, cétacé. Le descriptif qui suit est assez représentatif des paradonnées accompagnant désormais nos levés $2 \mathrm{D}$ et $3 \mathrm{D}$ : elles renseignent contours et enlèvements de matière, donnent les relations d'antéro-postériorité entre signes, pondèrent les interprétations des lectures brutes en surface de la roche (fig. 9). 
Figure 9

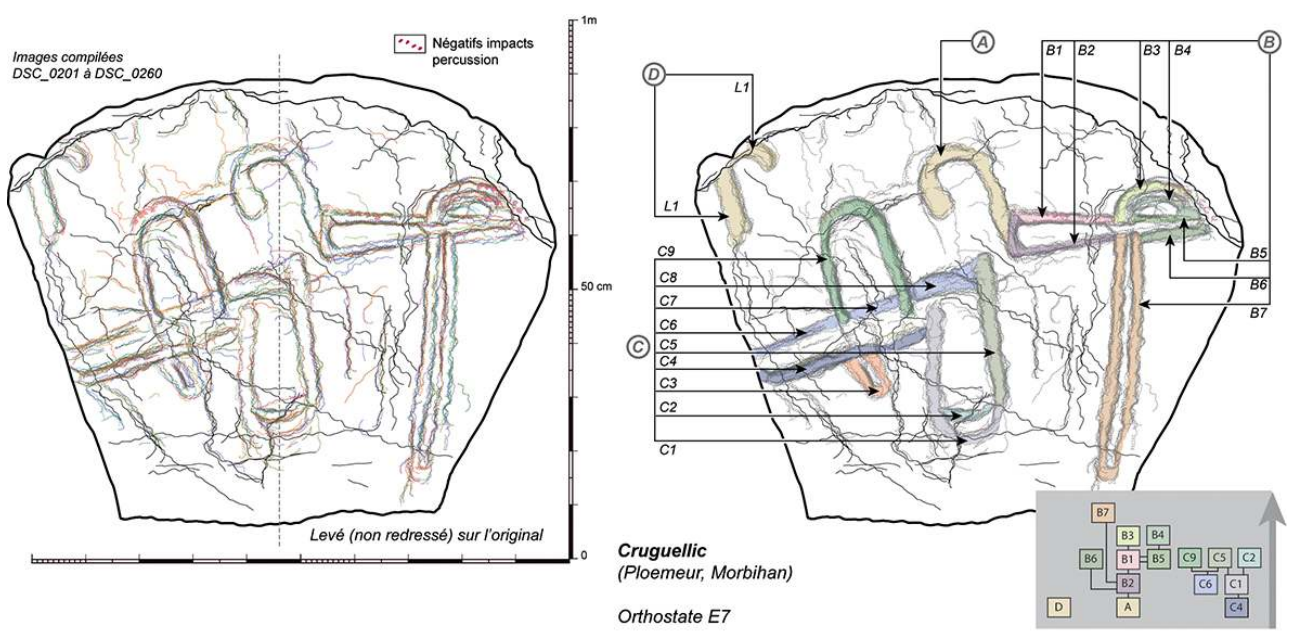

Synthèse des contours extraits des clichés sous éclairages obliques; synthèse des enlèvements de matière et dénomination des signes décrits ; matrice des relations inter-signes.

(c) Serge Cassen, Valentin Grimaud.

Le motif A est constitué d'un seul tracé en continu, le signe se confondant par conséquent avec le motif. Il s'agit d'une crosse senestre, penchée par rapport à un axe central et longitudinal proposé dans la configuration restaurée actuelle, qui passerait d'ailleurs par son milieu.

Le motif $B$ est une hache emmanchée.

$\rightarrow$ B1 et B2 dégagent la lame rectiligne, au tranchant étroit, B1 étant postérieur à B2. B2 recoupe nettement la crosse $\mathrm{A}$.

$5 \rightarrow$ B3 extrait par champlevé également le crosseron du manche proprement dit. B3 recoupe $\mathrm{B} 1$ et vient presque entamer le corps de la lame.

$\rightarrow$ B4 est l'enlèvement symétrique permettant de dégager le crosseron.

$\rightarrow$ B5 est un tracé exactement orienté dans le prolongement de B1. Il dessine le talon de la lame et semble recoupé par B4, mais la relation est fragile.

$\rightarrow$ B6 est l'enlèvement de matière symétriquement placé, sans rejoindre B5 semble-t-il, à moins que la bordure du support n'ait tronqué cette relation attendue.

$\rightarrow$ B7 est un tracé continu qui dessine le manche, recoupant B2 à gauche et B6 à droite. Notons que l'extrémité proximale est courbée, soit que le modèle l'exigeait, soit que la bordure de la stèle ne l'ait imposé.

40 Le motif $\mathrm{C}$ est sans discussion possible un des composants bien reconnaissable du registre néolithique de l'ouest de la France. Plutôt qu'une "hache-charrue», terme consacré, nous suggérons d'y voir un nouveau spécimen de cétacé (cachalot $\left.{ }^{15}\right)$, bien entendu fort simplifié par rapport aux figures plus réalistes de Locmariaquer et Sarzeau.

$41 \rightarrow \mathrm{C} 1$ dégage le côté gauche de la "tête", englobant d'ailleurs son extrémité, très endommagée.

$\rightarrow \mathrm{C} 2$ est une reprise, postérieure à C1. C'est un tracé courbe qui divise la surface en champlevé, rectiligne en haut, curviligne en bas. 
$43 \rightarrow$ C3 est un enlèvement placé sur le « ventre ", dans le prolongement de C9, mais qui lui est bien distinct. Le signe ainsi réservé est un segment rectiligne oblique.

$\rightarrow \mathrm{C} 4$ assure le tracé inférieur du "corps ». Il est interrompu par C1. Nous n'avons pas identifié son achèvement sur le bord de la stèle, où devrait se trouver la « queue ». le motif. Il s'agit cette fois d'une crosse dextre, mais interrompue par une cassure dans le support. Elle est penchée par rapport à un axe central et longitudinal. Le motif est excentré en regard de la composition en son ensemble, et surmonte ce qui devrait être l'emplacement de la queue du cachalot.

49 Un déroulé chronographique (en cinq phases) peut donc être déduit de ces relations d'antéro-postériorité, mais par économie, ne sera pas reproduit dans le cadre de cet article.

\section{La tranche nord de l'orthostate E7}

50 L'absence d'une représentation de "queue " à l'extrémité gauche du motif principal est finalement intriguante car elle serait une exception dans le répertoire actuel. La question s'est donc posée, à l'issue de la restitution des tracés, d'un éventuel prolongement du motif sur la tranche orientée au nord. Elle nous a conduit à revenir sur l'acquisition photogrammétrique en recalculant un modèle tridimensionnel spécifique et plus précis, sur la tranche, afin d'en faire ressortir le maximum de détails. Aucun enregistrement par éclairages tournants n'avait en effet été programmé, aucune gravure n'ayant été ici inventoriée par les fouilleurs.

51 Le bas de la figure 8 présente par conséquent le résultat de plusieurs illuminations virtuelles fournies par des filtres appliqués à la carte des normales. Il en ressort une anomalie en creux qui correspond exactement aux deux tracés détectés sur la face contiguë (face sud-est), sans pour autant que l'on puisse l'affirmer preuve à l'appui. Ces deux tracés supposés viennent s'achever sur une fissure naturelle dans le granite, fissure qui formerait alors la nageoire caudale dans notre hypothèse interprétative. Plus généralement, la formule s'apparente à un typique «hors-champ iconographique » qui entraîne une dynamique supplémentaire à l'image de l'animal en mouvement.

\section{La comparaison de l'orthostate E7 avec le levé antérieur}

Un seul levé graphique fut publié ${ }^{16}$, manifestement établi par calque transparent tendu sur un bloc inversé par rapport à la position actuelle ${ }^{17}$, résultat de la plus récente restauration. Les tracés suivent ensuite un parti pris de rectilignes, parfois effectives, parfois seulement fidèles aux fissures naturelles dans le granite. On reconnait bien entendu le manche de la hache sur la gauche et une moitié de la lame polie, mais qui sont autant de signes non identifiés par nos collègues (voir fig. 7). Un bord de la tête du cétacé 
est conforme à notre levé mais le souffle est arrêté sur une fissure, et repéré sur un seul côté. Bref, il était impossible à partir de cet enregistrement de reconnaître les présents motifs, et quoi qu'il en soit, « l'écusson » décrit par la littérature est totalement absent.

\section{Résumé conclusif}

S'ajoutant au géométral de l'architecture funéraire, le site de Cruguellic donne l'occasion d'un descriptif de monolithes gravés, restes probables d'anciennes stèles en réemploi. Notre attention s'est portée sur E7 où les résultats sont sans commune mesure avec le précédent levé. Pour cet article, l'inventaire des désordres se limitera d'ailleurs à ce support orné, où la coïncidence notée entre une concentration de lichens et la partie très dégradée de l'extrémité céphalique du motif de cétacé n'est sans doute pas le simple reflet du hasard (fig. 10). Soulignons que la colonisation biologique s'est faite en 42 ans, cette partie du bloc ayant été trouvée enterrée en 1974.

Figure 10

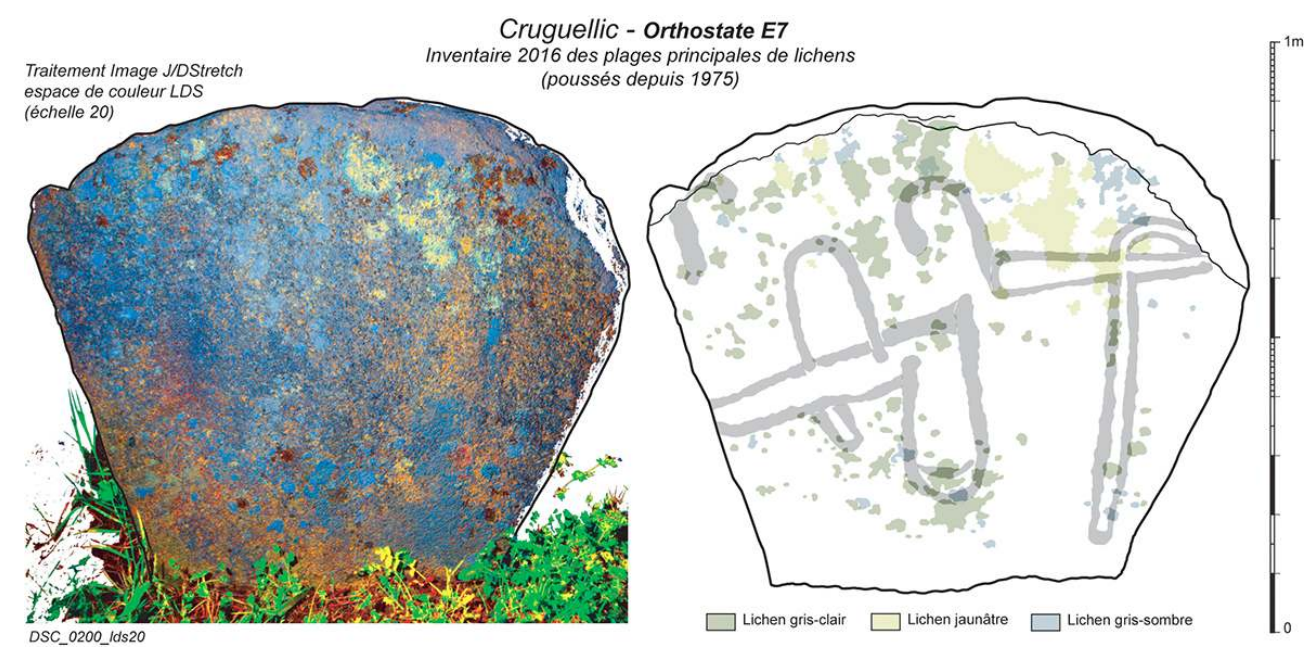

Décorrélation d'un cliché numérique dans l'espace de couleur LDS (traitement ImageJ / DStretch) pour une mise en évidence des colonisations biologiques ; cartographie des lichens.

(c) Serge Cassen, Valentin Grimaud.

Nous ne saurions trop insister sur le grave risque d'altération qu'encourent les deux dalles gravées, directement exposées aux agents atmosphériques, mais aussi objets d'une pression touristique (depuis plusieurs années, un festival utilise une scène aménagée sur une partie du cairn pour des animations - conteurs, musiques). La publication même de cet article dans In Situ va rendre... publique la présence insoupçonnée de telles gravures, et l'on peut donc s'attendre à une légitime curiosité à leur endroit, au risque parfois de surlignages colorés dommageables. La possibilité de conserver ces deux dalles dans un espace muséal de type lapidaire, tandis que des fac-similés prendraient leur place (et notamment en rehaussant de teintes les gravures désormais comprises), nous apparait comme une priorité conservatoire, un projet tout à fait réalisable. 


\section{Synthèse}

55 Notre démarche articule, en résumé, l'exigence d'une recherche fondamentale au sein d'un processus conservatoire, tout en préparant l'objet archéologique à sa valorisation auprès des publics.

$56 \rightarrow L a$ composante recherche assure l'environnement scientifique des processus d'enregistrements et de traitements des données devant aboutir à une classique information archéographique (plans, élévations, programme iconographique).

$57 \rightarrow$ La composante conservatoire permet non seulement une aide à la décision quant aux nouvelles mesures de protection et de présentation dont ces monuments pourraient bénéficier (état des lieux cartographié), mais assure la pérennité des données sauvegardées suivant des formats exploitables à très long terme, sans perte d'informations. Cet objectif est atteint grâce au logiciel aLTAG3D ${ }^{18}$ développé par le Consortium 3D SHS.

$58 \rightarrow$ La composante médialogique propose des visuels interprétés et interprétables, en suggérant différentes possibilités de restitution de l'objet patrimonial, à charge pour les élus et les administrations de les développer (modèles pour animation 3D, en accès libre).

59 Du terrain au laboratoire, de l'image enfouie à l'image restituée, l'archéologie est un de ces lieux du savoir où l'étendue des possibilités offertes par les technologies dites numériques devrait en théorie pouvoir pleinement s'appliquer. À cet égard, les architectures funéraires et symboliques du Néolithique se prêtent bien à ce désir immédiat de figurer des objets très visibles. Il semble si aisé de se les re-présenter puisqu'on les touche des mains, puisqu'on les évalue à leur juste puissance évocatrice. Mais dans «immédiat », il y a par définition absence de médiation, d'où ce risque de ne reproduire qu'une image d'Épinal, de restaurer selon une image préconçue ${ }^{19}$, de ne pas tenir compte de la nuance...

$60 \rightarrow$ Le dolmen de Cruguellic nous fait passer de l'analogique au numérique, après une restauration in situ du tumulus et des espaces funéraires documentés par la fouille; à nous de proposer l'autre terme de l'alternative : restitutif, réversible, actualisable.

$61 \rightarrow$ Le dolmen de la Planche à Puare vous propose de passer du numérique au digital, en déposant votre doigt sur l'écran pour soulever ces dalles, le grand rêve des humains en lutte contre la gravité. Pourtant, seule la fouille du tumulus permettra de connaitre la géométrie de l'enveloppe afin d'en proposer la restauration virtuelle.

62 Bien sûr, nous jouons sur les mots, le digital français ("qui appartient aux doigts») n'étant pas superposé au digital anglais ("qui utilise des nombres»). Des agences de communication françaises ont en fait trouvé plus facile d'utiliser le même mot dans les deux langues... Il y a plusieurs décennies maintenant, la civilisation des médias, des « mass media », se définissait déjà par le rejet (agressif) de la nuance ${ }^{20}$. Mais la différence entre numérique et digital est plutôt une tension, non un binarisme ${ }^{21}$; deux mots qui introduisent des nuances que ni la poésie ni la pensée n'iront reprocher à la sémantique... 


\section{NOTES}

1. - L'HeLGOUAC'H, J. Les Sépultures mégalithiques en Armorique. Rennes: Université de Rennes, 1965.

2. - CASSEN, Serge, LESCOP, Laurent, GRIMAUD, Valentin, ROBIN, Guillaume. " Complementarity of acquisition techniques for the documentation of Neolithic engravings: lasergrammetric and photographic recording in Gavrinis passage tomb (Brittany, France) ». Journal of Archaeological Sciences, 2014, 45, p. 126-140.

3. - VOLKOV, Théodore [VOVK, Fedir]. «Dolmens de l'île d'Yeu». Bulletin de la Société d'anthropologie de Paris, 1896, t. VII, 3, p. 241-246.

4. - Géoréférencement par satellite.

5. - AUGER, Augustin. "Les fouilles du dolmen de la Planche à Puare à l'île d'Yeu (Vendée) ». A nnuaire de la Société d'émulation de la Vendée, 1884, 4, p. 75-84, p. 3.

6. - Ibid., p. 3.

7. - Ibid., p. 3.

8. - BAUDOUIN, Marcel. «Découverte de dents de cachalot dans un dolmen de l'île d'Yeu (Vendée) ». Bulletin de la Société préhistorique française, 1907, t. 4, 2, p. 120-122.

9. - BAUDOUIN, M. « L'ossuaire à os décarnisés et brisés de l'allée couverte de la Planche à Puare, à l'île d'Yeu (Vendée) ». Bulletins et Mémoires de la Société d'anthropologie de Paris, 1914, VI ${ }^{\mathrm{e}}$ Série, t. 5, fasc. 2, p. 98-123.

10. - L'HELGOUAC'H, J. Mégalithes en Loire-Atlantique: recherches récentes autour de l'estuaire de La Loire. Nantes : Association d'études préhistoriques et historiques des Pays de La Loire, 1986.

11. - LE ROUX, Charles-Tanguy. « Le mobilier du dolmen de Cruguellic en Ploemeur (Morbihan)». Bulletin de la Société préhistorique française, 1978, t. 75, n 9, p. 281-285.

12. - LE ROUX, Ch.-T., LECERF, Yannick. « Le dolmen de Cruguellic en Ploemeur (Morbihan) et les sépultures mégalithiques transeptées armoricaines ». Dans L'Architecture mégalithique. Colloque du $150^{e}$ anniversaire de la Société Polymathique du Morbihan. Vannes : Château-Gaillard, 1977, p. 143-160.

13. - Ibid.

14. - LE ROUX, Ch.-T. « Bretagne ». Gallia préhistoire, 1975, t. 18, fasc. 2, p. 511-539.

15. - CASSEN, Serge, VAQUERO LASTRES, Jacobo. «La Forme d'une chose ». Dans CASSEN, Serge, BOUJOT, Christine et VAQUERO, Jacobo (dir.). Éléments d'architecture. Exploration d'un tertre funéraire à Lannec er Gadouer (Erdeven, Morbihan). Constructions et reconstructions dans le Néolithique morbihannais. Propositions pour une lecture symbolique. Chauvigny: Association des publications chauvinoises, 2000, p. 611-656.

16. - LE ROUX, Ch.-T., LECERF, Y. «Le dolmen de Cruguellic en Ploemeur (Morbihan) et les sépultures mégalithiques transeptées armoricaines ». Art. cit.

17. - LE ROUX, Ch.-T. « Bretagne ». Gallia préhistoire, Art. cit., p. 511-539.

18. - Voir le site : http://altag3d.huma-num.fr [consulté le 01/02/2018].

19. - CASSEN, S., LESCOP, L., GRIMAUD, V. «Pour une critique de la représentation tridimensionnelle des architectures mégalithiques en Europe occidentale. Méthodes et usages actuels ». Annales de Bretagne et des Pays de l'ouest, 2013, t. 120, 1, p. 7-31.

20. - BARTHES, Roland. La Préparation du roman. Cours au Collège de France 1978-1979 et 1979-1980. Paris : Éd. du Seuil, 2015.

21. - ROPARS, Fabian. "Faut-il dire numérique ou digital ?». Blog du modérateur - Le média des professionnels du digital, 2015. Voir le site: https://www.blogdumoderateur.com/numerique-oudigital/ [consulté le 11/01/2018]. 


\section{RÉSUMÉS}

Durant le Néolithique, l'ouest de la France se caractérise par le développement d'une monumentalité funéraire et la construction de vastes ouvrages de stèles, remarquables par le fait que ces objets archéologiques sont le plus souvent conservés en élévation. Cette singularité fut aussi la cause de leur destruction, notamment à partir du XIX ${ }^{\mathrm{e}}$ siècle. Si la représentation tridimensionnelle de ces architectures anciennes fut assez tôt une préoccupation de quelques explorateurs, le développement récent des outils numériques a permis de mieux établir les fondements de leur documentation à travers un géométral rigoureux. La présence de signes gravés sur certains monolithes ajoute une difficulté supplémentaire en termes d'échelles d'observation et de rendus, difficulté désormais surmontée par la complémentarité de différentes techniques d'acquisition. Dès lors, ces nouvelles représentations architecturales posent la question des restaurations qui ont affecté dès la fin du XIX siècle, et continuent à toucher, nombre de ces dolmens et autres stèles. Les restitutions virtuelles autorisent de nouvelles configurations, évidemment réversibles et bien plus didactiques. Notre contribution prendra donc pour exemple deux tombes monumentales, dites transeptées, d'un type bien particulier sur le littoral atlantique, l'une dans le Morbihan, l'autre en Vendée, enregistrées en 2D et 3D selon les mêmes protocoles. L'une vient de révéler de nouvelles gravures inédites au sein d'un site restauré et figé ; l'autre, menacée par la montée du niveau des mers, sera prochainement mise en valeur à travers un dispositif de découverte virtuelle sur écran tactile.

During the Neolithic era, the West of France was characterised by the development of monumental funerary structures and the erection of vast ensembles of steles. These are remarkable as archaeological objects since, more often than not, they are preserved in an upright position This characteristic was also the cause of their destruction, in particular from the nineteenth century. The three-dimensional representation of these ancient architectural sites was undertaken by certain early explorers but the recent development of digital tools has made it possible to establish more reliable documentation using rigorous geometric plans. The presence of engraved signs on some monolithic elements can add additional difficulties in terms of the scales of observation and of renderings, but these difficulties can now be overcome using different but complementary techniques of data acquisition. These new architectural representations raise the issue of the restoration of the ensembles, a question already raised at the end of the nineteenth century and which continues to arise where these dolmens and steles are concerned. Our contribution will take a look at two monumental tombs of a specific 'transepted' type, located near the Atlantic coast, one of them in the Morbihan department and the other in the Vendée. The two tombs have been recorded in 2D and 3D according to the same protocols. One has just revealed new engravings within a site that had already been restored. The other, which is threatened by the rising sea level, will be interpreted using a virtual display on a touch screen. 
INDEX

Keywords : Neolithic, megalith, geometric plans, iconography

Mots-clés : Néolithique, mégalithe, géométral, iconographie

\section{AUTEURS}

\section{SERGE CASSEN}

Laboratoire de recherches en archéologie et architectures (UMR 6566), directeur de recherche CNRS, Université de Nantes serge.cassen@univ-nantes.fr

\section{VALENTIN GRIMAUD}

Laboratoire de recherches en archéologie et architectures, architecte et ingénieur d'étude, Université de Nantes valentin.grimaud@univ-nantes.fr 